\title{
Globe
}

Revue internationale d'études québécoises

\section{L'intrigue de la production moderne du religieux au Québec}

\section{Robert Mager et E.-Martin Meunier}

Volume 11, numéro 1, 2008

La religion au Québec. Regards croisés sur une intrigue moderne

URI : https://id.erudit.org/iderudit/1000488ar

DOI : https://doi.org/10.7202/1000488ar

Aller au sommaire du numéro

Éditeur(s)

Globe, Revue internationale d'études québécoises

ISSN

1481-5869 (imprimé)

1923-8231 (numérique)

Découvrir la revue

Citer ce document

Mager, R. \& Meunier, E.-M. (2008). L'intrigue de la production moderne du religieux au Québec. Globe, 11(1), 13-20. https://doi.org/10.7202/1000488ar d'utilisation que vous pouvez consulter en ligne.

https://apropos.erudit.org/fr/usagers/politique-dutilisation/ 


\title{
INTRODUCTION.
}

\author{
L'INTRIGUE
}

\section{DE LA PRODUCTION MODERNE DU RELIGIEUX AU QUÉBEC}

\author{
ROBERT MAGER \\ Université Laval \\ E.-MARTIN MEUNIER \\ Université d'Ottawa
}

La religion n'est pas un objet culturel comme un autre, qui puisse être abordé avec détachement, surtout au Québec. Ceux qui l'étudient depuis longtemps le savent bien: s'intéresser à la religion, c'est s'exposer à la suspicion de ceux qui la tiennent pour dépassée et exsangue, ou la considèrent comme une réalité dont on aurait trop parlé et qu'il faudrait taire, en attendant qu'elle s'éteigne d'elle-même'.

Puisque les raisons de ce discrédit sont multiples et profondes, il importe, plus encore pour le lectorat international, de les situer. Elles relèvent autant de l'essor d'une nouvelle structure sociohistorique fondamentale ayant trait à la genèse et au déploiement du monde moderne et de ses suites ${ }^{2}$, que de certaines dérives de la religion elle-même (cléricalisme, autoritarisme, centralisme, etc.) touchant ce que le sociologue Fernand Dumont nommait à juste titre l' "institutionnalisation" du religieux ${ }^{3}$. Au Québec, la critique de la religion prit des proportions importantes, assez pour que certains parlent d'elle comme d'une «colère

$$
++
$$

1. Jacques Grand'Maison évoque cette expérience dans l'introduction de son ouvrage Pour un nouvel humantsme (Montreal, Fides, 2007).

2. Michel FREITAG, "La dissolution post-moderne de la reférence transcendantalen, Cahrers de recherche sociologique, n॰ 33, 1999, p. 181-217. Voir aussi, du même auteur, Dralectique et soctité. Tome 2. Culture, pouvoir, contrôle. Les modes de reproductzon formels de la societté, Montréal, Édicions coopératives Albert Saint-Martin, 1986.

3. Fernand DUMONT, Linstitution de la théologie. Essai sur la struation du théologien, Montréal, Fides, 1987. 
antithéologique ${ }^{4}$ ". Elle répondait, en un sens, à une période de forte emprise de l'institution catholique sur la culture et les institutions sociales, du milieu du XIX ${ }^{e}$ siècle au milieu du XXe siècle, juste avant la Révolution tranquille ${ }^{5}$. Si celle-ci a comporté un ensemble de transformations sur de nombreux plans (économique, politique, social, culturel, etc.), on l'a rapidement interprétée comme une émancipation de la société québécoise par rapport à cette ère dite de "Grande noirceur" $"$ ".

Pour plusieurs de ses protagonistes, et sans doute plus encore pour la génération qui suivra, cette révolution sociale et culturelle signait l'entrée dans une ère moderne ${ }^{7}$, qu'on définissait sinon contre la religion, du moins contre une certaine vision traditionnelle du catholicisme ${ }^{8}$. À partir d'idéaux et de principes jugés nouveaux ${ }^{9}$, souvent au nom de légitimités scientifiques en émergence ${ }^{10}$, plusieurs revendiquaient une transformation en profondeur de la société québécoise. C'est à cette époque que les sciences humaines et sociales se sont développées massivement à l'intérieur d'un réseau universitaire en pleine expansion ". S'amorçaient dès lors un processus de définition utopique d'un Québec nouveau à construire et la liquidation d'un passé religieux, qui s'est faite sans ménagement. Dans ce procès, ces sciences

$$
+\div
$$

4. Gilles LABELLE, "Sens et destın de la colère antithéologıque au Québec après la Révolution tranquille », Alain G. GAGNON, J. PALLARD et B. GAGNON [dir.], Deverstés et identités au Quebec et dans les régons d'Europe, Bruxelles/Québec, Presses interunıversitaures européennes - Peter Lang/Presses de l'Université Laval, 2006, p. 337-364. Voir aussı, du même aureur, *Psésence du "problème théologico-politique" dans la pensée politique de la Révolution tranquille: Pierre Vadeboncoeur, l' "autorité du peuple" et la "re-spiritualisatıon" de la socıété québécoisen, E.-M. MEUNIER et J. Y. THÉRIAULT [dır.], Les impasses de la mémoire. Histoire, fliation, nation et religzon, Montréal, Fides, 2007.

5. Jean GOULD, "La genèse catholique d'une modernisatıon bureaucratıque", Stéphane KELLY [dir.], Les idees mènent le Québec Essat sur une senstbiltté hustorıque, Saince-Foy (Québec), Presses de l'Université Laval, 2003, p. 146-174.

6. Jocelyn LETOURNEAU, "La Révolunion tranquille, catégorie identitaire du Québec contemporaın "Alain G. GAGNON et Michel SARRA-BOURnet [dır.], Duplessis. Entre la Grande noirceur et la soctété libérale, Montréal, Québec Amérique, 1997, p 175-209; E.-Martin MEUNIER, "La "Grande Noirceur" en tons de gris. une mémoire mitoyenne est-elle possible?», Jean-Phulippe WARREN [dir.], Le Québec en conq cltchés. Canq grands mythes québécois revistués, Québec, Nota bene, à parâtrre au printemps 2008.

7. Marcel FOURNIER, L'entrée dans la modernité science, culture et société au Québec, Montréal, Éditions Albert SaintMartın, 1986.

8. E.-Martin MEUNIER et Jean-Philuppe WARREN, Sortur de la "Grande notrceur". Lhorzzon personnaltste de la Revolution tranquille, Sillery, Édıtions du Septentrion, 2002; Louise BIENVENUE, Quand la jeunesse entre en scène. LAction catholuque avant la Révolutzon tranquille, Montréal, Boréal, 2003.

9. Idéaux et principes qui ont puisé tantôt dans le personnalısme chrétien (notamment pour la génération de la revue Cité libre), rantôt dans un socialisme décolonisateur (pour la génétation de la revue Parti Prts). Voir nocamment Stéphanie ANGERS et Gérard FABRE, Echanges intellectuels entre la France et le Québec 1930-2000. Les réseatux de la revue Esprit avec La Relève, Ciré libre, Partı prıs et Possıbles, Québec, Presses de l'Unıversité Laval, 2004 ; et, bien sûr, l'ouvrage classique d'André J. BÉLANGER, Ruptures et constantes: quatre idéologies du Quebec en élatement: La Relève, la JEC, Cité libre, Parti pris, Montréal, Hurtubise HMH, 1977.

10. Jean GouLD, Des bons pères aux experts. Les elites catholiques et la modernusation du systeme scolatre au Quebec, 19401964, Sante-Foy, Presses de l'Université Laval, 1999.

11. Jean-Charles FALARDEAU, L'essor des sctences sociales au Canada françats, Québec, Minıstère des affaires culturelles, 1964; Jean-Philippe WARREN, L'engagement sociologigue la tradition sociologique du Quebec francophone, 1886-1955, Montréal, Boréal, 2003; Luc CHARTRAND, Raymond DUCHESNE et Yves GiNGRAS, Histotre des sctences au Quebec, Montréal, Boréal, 1987. 
n'étaient pas neutres: souvent nées d'un arrachement aux perspectives et au contrôle d'une conception traditionnelle de la religion, elles incitaient leurs représentants à en déclarer la mort ou à en faire l'autopsie. Il est notable de constater que l'interprétation "Grande noirceur/Révolution tranquille» fut produite très rapidement après l'événement, et parfois même par les acteurs et penseurs du changement social, désireux de donner sens et impulsion à leurs réformes. Un schéma directeur s'imposa rapidement, schéma qu'on pouvait repérer dans toutes sortes d'oppositions typologiques telles folk society/urban society (Hughes) ou société traditionnelle/société technologique $(\text { Dumont })^{12}$. Une sorte de nouveau paradigme national était né: la question de la Révolution tranquille allait être enserrée dans le schéma tradition/ modernité et, dans ce procès sociohistorique, le fait religieux, quel qu'il soit, allait peu à peu être rapporté au vecteur unique de la tradition dont il fallait désormais se débarrasser ${ }^{13}$. Cette façon de concevoir le Québec moderne sorti et "dépris" de sa chape de plomb cléricale - allait profondément marquer aussi bien l'imaginaire des Québécois ${ }^{14}$ que les formes de transmission de normes et de valeurs ${ }^{15}$. Un rapport trouble à la religion semblait s'instaurer: même s'intéresser au passé religieux ou à l'économie contemporaine du religieux devenait suspect.

Dans le Québec des années 1970 et 1980 , il était mal vu de s'intéresser à la religion, comme pourraient en témoigner les quelques sociologues, historiens et théologiens qui s'y adonnèrent néanmoins. Or il semble bien que cette quarantaine soit finie. Plus de quarante ans après la Révolution tranquille, un ensemble de facteurs contribuent à attirer l'attention sur les questions religieuses et à renouveler le regard. D'abord, la disparition annoncée ne s'est pas produite: il y a bien eu quelque chose comme une "sortie de la religion", mais celle-ci n'a pas signifié la fin de la religion ${ }^{16}$. On observe plutôt des persistances ${ }^{17}$, des déplacements ${ }^{18}$, des

$$
++\div
$$

12. Everect C. HưgHES, Rencontres de deux mondes, Montréal, Boréal express, 1972; Fernand DumONT, Le lieu de l'bomme. La culture comme distance et mémoire, Montréal, Édition HMH, 1971.

13. Pour une explicitacion du rapport des Québécois à la relıgion lors de la pértode post-Révolution tranquille, voir E.-Marcin MEUN1ER, "Une nouvelle sensibilité pour les "Enfants du concile"? ", Stéphane KELLY [dir.], op cit., p. 93-106. 14. Raymond LEMIEUX er Jean-Paul MONTMINY, Le catholzcisme quebecois, Québec, Éditions de l'Institut québécois de recherche sur la culture, 2000.

15. Roberc MAGER, "La transmission de la religion ", Jean-Marc LAROUCHE et Guy MÉNARD [dir.], L'túde de La religion au Québec. Bulan et prospectuve, Québec, Presses de l'Universıté Laval, 2001.

16. Marcel GAUCHET, Le desenchantement du monde. Une histoire polstigue de la religzon, Paris, Gallimard, 1985; Robert MAGER, "Secularization in Quebec: Phenomenon, Expression and Theory", W. GRAB [dir.], Secularization Theortes, Religuous Identıty and Practical Theology, Münster (Allemagne), LIT Verlag, à paraître en 2008.

17. Raymond LEMIEUX, *Le catholicisme québécois: une question de culture», Sociologre et socittés, vol. 22, $n^{\circ} 2$, octobre 1990, p. 145-164.

18. Roland CHAGNON, "Religion, sécularisation et déplacements du sacré», Sciences religzeuses/Studzes in Religion, vol. $18, n^{\circ} 2$, printemps 1989, p. $127-151$. 
transformations ${ }^{19}$, voire des résurgences ${ }^{20}$, au point où le chantre de la sécularisation, le sociologue Peter L. Berger, a changé son fusil d'épaule et fait l'annonce d'un "réenchantement du monde ${ }^{21}$ ». Plus prudents, d'autres sociologues préferent parler d'une "recomposition du champ religieux ${ }^{22}$ ". Ainsi, si la religion fait un "retour", ce n'est pas tant dans les dynamiques sociales elles-mêmes qu'à l'avant-scène de l'actualité, à la faveur, notamment, des grands bouleversements qui ont suivi les événements de septembre 2001. Quel que soit son rôle effectif dans les jeux sociopolitiques et militaires actuels, la religion apparaît comme un facteur incontournable auquel il importe de réfléchir. Enfin, au Québec même, le recul historique permet à cette réflexion de se déployer dans un climat plus serein: il devient possible de se pencher sur des questions aussi diverses que l'apport du catholicisme à la constitution de la société québécoise, l'aménagement de la pluralité religieuse, la disposition du patrimoine religieux ou la contribution des religions à une éthique publique. En fait, un examen rapide des débats des dernières années fait apparaître la diversité des domaines touchés par la religion : les nouvelles productions religieuses, les questions de morale sexuelle, l'enseignement religieux à l'école, l'intérêt multiforme pour la "spiritualité", le port d'insignes religieux dans l'espace public, les accommodements raisonnables, etc.

Il y a, en ce sens, une "intrigue moderne ${ }^{23}{ }^{\prime}$. Alors que la modernité était censée procéder du retrait du religieux, elle ne paraît pas seulement devoir s'en "accommoder", mais semble plutôt donner lieu à des dynamiques religieuses originales et encore peu élucidées. La sociologue Danièle Hervieu-Léger en est ainsi venue à parler de "productions religieuses de la modernité24 ". Dans la même veine, le philosophe et historien Marcel Gauchet indique que le projet même d'autonomie moderne appelle en creux des ressources de sens que les religions (mais pas seulement elles) peuvent

$$
+4
$$

19. Yvon DESROSIERS [dir.], Religton et culture au Québec: figures contemporaines du sacré, Montréal, Fides, 1986.

20. Danièle HeRVIEU-LEEGR, Le pelerin et le converts. La religion en mouvement, Paris, Flarnmarion 1999.

21. Peter L BERGER [dir.], Le reenchantement du monde, Parıs, Bayard, 2001.

22. Raymond LEMIEUX, "Notes sur la recomposition du champ religieux", Sciences religreuses/Studies in Religzon, vol.25, $\mathrm{n}^{0} 1,1996, \mathrm{p}$ 61-86.

23. Le terme "modernité" est entendu ici au sens large Sans entrer dans le vaste débat sur la question de l'appellation du type de sociécé contemporaine (modernuté avancée, post-modernıté, hypermodernıté, ultramodernité), ll s'agit avant tout de sıgnaler une intrıgue qui survient avec la rupture moderne et sa suıre. Voir, sur ce débat, l'idée de socıété "posttradıtionnelle* que l'on retrouve chez Yves BONNY, Sociologie du temps présent, Parıs, Armand Colin, 2004.

24. Danièle HERVIEU-LEGER, Vers un nouveau christianisme? Introductzon a la soctologze du chrstrantsme occtdentah Paris, Cerf, 1986. L'auteure a peaufiné cette idée dans plusieurs autres écnts, nocamment : La religson pour mémotre, Paris, Cerf, 1993 ; "Productions religieuses de la modernıté : les phénomènes du crorre dans les sociétés modernes ", Brigıtte CAULJER [dır.], Religion. sécularisation, modernité. Les expértences francophones en Amérique du Nord, Sainte-Foy (Québec), Presses de l'Universicé Laval, 1996, p. 37-58; La religion en miettes ou la question des sectes, Paris, Calmann-Lévy, 2001. 
mettre en œuvre dans l'espace public ${ }^{25}$. Près d'un demi-siècle après la Révolution tranquille, il n'est donc plus tant question d'être pour ou contre la religion que de chercher à comprendre comment elle évolue en modernité et, inversement, comment la modernité - de par sa constitution et ses contradictions internes - semble lui être un terreau fertile ${ }^{26}$.

Il y a là de multiples chantiers déjà très actifs. En ce sens, ce numéro de Globe arrive à point nommé. Consacré à "la religion au Québec", il embrasse large et a dû prendre la forme d'un numéro double. Nous n'avons pas voulu restreindre a priori le champ du questionnement: il s'agissait de permettre à un ensemble de réflexions en cours de se manifester. Nous avons procédé à la fois par un appel à communications et par des invitations ciblées. Nous avions d'abord l'ambition de montrer le renouvellement des perspectives, d'indiquer comment la religion au Québec fait actuellement l'objet de nouvelles lectures concernant l'origine de la Nouvelle-France, la supposée Grande noirceur du Québec d'antan ou la refondation du Québec moderne. Mais les textes effectivement reçus nous ont entraînés dans d'autres directions. Presque tous consacrés au Québec moderne, ils ne cherchent pas tant à infirmer les jugements d'hier qu'à comprendre les dynamiques actuelles, qu'il s'agisse du rapport au passé religieux, de l'aménagement de la pluralité religieuse ou de l'évolution du catholicisme.

En procédant ainsi, et malgré l'espace d'un numéro double, nous avons dû renoncer à traiter de tous les sujets qui mériteraient l'attention. Ce numéro n'a donc rien d'un manuel. Il ne s'y trouve pas de contribution sur des sujets clés comme les religions autochtones, la fondation religieuse de la Nouvelle-France, la période 1840-1950, le judaïsme ou le patrimoine religieux. En revanche, les articles retenus explorent de façon originale plusieurs aspects de cette réalité multiforme du religieux. Ils le font à partir d'une pluralité de perspectives disciplinaires: sociologie, histoire, philosophie, études littéraires, théologie. À travers ces regards croisés, c'est la complexité et l'évolution du champ religieux qui apparaissent, intimement liées à celles de la modernité québécoise elle-même.

D'entrée de jeu, plusieurs articles traitent du rapport du Québec moderne à son passé religieux. Le philosophe Daniel Tanguay examine deux œuvres majeures contemporaines, la pièce de théâtre Bureaux,

$+\div$

25. Marcel GAUCHET, La religion dans la democratic. Parcours de la latcuté, Paris, Gallimard, 1998, p. 104 er suivantes. 26. Roberr MAGER, * Pour l'amour du monde. Remarques sur l'expérience de la transcendance dans la modernıté avancée n, P. GAUDETTE [dir.], Mutations culturelles et transcendance. A l'aube du XXX sizcle, supplémenc de Laval theologigue et philosophique, 2000, p. 145-161. 
d'Alexis Martin, et le film La Neuvaine, de Bernard Émond. Chacune d'elles témoigne de l'inquiétude spirituelle multiforme du Québec "postcatholique». À l'encontre du tournant thérapeutique effectué par la religion ellemême, l'œuvre d'art relance la question de la transcendance, dont les voies demeurent cependant à trouver. L'article du philosophe Gabriel Malenfant s'inscrit dans la foulée de ce questionnement: dans un Québec où la religion s'est effacée, est-il possible d'envisager une réappropriation "laique» du religieux? La nécessité de développer une éthique commune le conduit à explorer les possibilités ouvertes en ce sens dans l'œuvre tardive du philosophe juif Hermann Cohen. Dans une autre perspective, mais toujours en lien avec le problème de la mémoire, le littéraire Pierre Rajotte et le sociologue Jacques Caroux se penchent sur un ensemble de récits que des Québécois ont faits de leur pèlerinage à Compostelle. De la multiplicité des parcours et des expériences se dégage la nécessité de tracer un itinéraire spirituel personnel, à même un héritage religieux exploré concrètement, dans l'espace et le temps de la marche. Leur article donne lieu à un examen très fin du rapport trouble des Québécois par rapport à la religion et au catholicisme. La contribution de l'historien Éric Bédard, pour sa part, poursuit dans la même veine en ciblant explicitement le rapport au passé mis en scène par une série de films de genre historique produits dans les années 2000: Séraphin. Un homme et son péché (2003), Le Survenant (2005) et Aurore (2005). Ces trois films reprennent des "classiques» de la culture québécoise en y accentuant le caractère oppressif de la religion, alimentant ainsi le souvenir d'une "Grande noirceur " passée dont la modernité québécoise constituerait le contrepoint.

Les articles suivants portent sur la question de la laïcité québécoise. Le sociologue Mathieu Bock-Côté propose une lecture décapante du débat sur les accommodements raisonnables, dans lequel il voit une crise du multiculturalisme à la québécoise. Le sociologue David Koussens, pour sa part, compare les manières dont la société française et la société québécoise ont traité la question du port de signes religieux en public, ces politiques différentes traduisant des aménagements différents de la laïcité.

Trois articles sont consacrés à la situation de l'Église catholique dans le Québec moderne. La théologienne Anne Fortin effectue un survol des positions de l'Église depuis les années 1960 et interroge les conditions qui lui permettraient de retrouver une pertinence dans l'espace public. Analysant un ensemble de sondages qu'il a menés lui-même depuis trente ans, le sociologue Reginald W. Bibby remet en question la perception d'une fin du catholicisme québécois. Il juge persistantes les attentes religieuses de la 
population catholique; le problème se situerait largement, selon lui, du côté de l' "offre", c'est-à-dire de la capacité et de la volonté de l'Église de répondre à ces attentes. Les théologiens Guy Jobin et Isabelle Archambault, pour leur part, examinent les positions de l'Eglise relativement à l'homosexualité, notamment dans le dossier des unions homosexuelles; ils montrent l'impact de la modernité avancée sur l'argumentation théologique elle-même.

Les deux derniers articles traitent de la rencontre entre des religions du monde et la modernité québécoise. L'historien Mouloud Haddad montre comment le soufisme s'implante au Québec, en s'adaptant à la réalité locale et en tirant profit des nouvelles technologies pour favoriser, par le moyen d'Internet, la constitution d'une communauté virtuelle des croyants. L'historien Louis Rousseau rend compte des manières dont des immigrants d'origine africaine composent avec leur religion pentecôtiste et la sécularité de leur société d'accueil pour réaliser leur nécessaire intégration.

Avec Louis Rousseau, un numéro comme celui-ci se devait de donner la parole à deux autres fondateurs des études religieuses québécoises. D'une part, la chronique "Perspective" a été confiée au sociologue des religions Raymond Lemieux, récipiendaire du Prix André-Laurendeau de l'ACFAS en 2001. Dans ce texte, Raymond Lemieux trace un portrait de l'évolution de la religion dans le Québec moderne, où l'on voit que les institutions religieuses tendent à céder le pas aux trajectoires diverses de sujets en quête de sens. D'autre part, une première "note critique" est rédigée par l'historien Michel Despland, qui examine cinq livres significatifs des recherches récentes sur la religion au Québec. Une seconde "note critique», proposée par le sociologue Jean-Philippe Warren, s'intéresse plus spécifiquement aux travaux de jeunes chercheurs qui jettent un regard neuf sur l'histoire du catholicisme québécois.

De l'ensemble de ces textes se dégage une impression très nette: l'évolution de la religion au Québec est intimement liée à l'évolution d'ensemble de la société québécoise. Loin d'être un épiphénomène, la religion continue de hanter cette évolution. Marcel Gauchet écrivait à ce sujet:

Si peu visible que ce soit, la place et le statut de la religion sont au cœur des transformations présentes de la démocratie [...]. Bien comprendre les transformations considérables qui sont en train d'affecter la substance intime des démocraties et leur intelligence d'elles-mêmes [...] exige de prendre en compte la dimension religieuse. Dans l'autre sens, les changements très profonds que subit 
la religion [...] ne deviennent pleinement intelligibles que si on les met en relation avec la dimension politique et sociale ${ }^{27}$.

Il y a là, pour le moins, une invitation à continuer de prêter attention aux entrecroisements de la religion et de la modernité, une intrigue dont le dénouement est loin d'être décidé. 